\title{
Cortical microstructure in primary progressive aphasia: a multicenter study
}

Ignacio Illán-Gala1,2,3*+ , Victor Montal ${ }^{1,2+}$, Sergi Borrego-Écija ${ }^{2,4}$, Maria Luisa Mandelli5 , Neus Falgàs ${ }^{3,5}$, Ariane E. Welch', Jordi Pegueroles 1,2, Miguel Santos-Santos ${ }^{1,2}$, Alexandre Bejanin ${ }^{1,2}$, Daniel Alcolea ${ }^{1,2}$, Oriol Dols-Icardo 1,2, Olivia Belbin 1,2, Ma . Belén Sánchez-Saudinós ${ }^{1}$, Nuria Bargalló ${ }^{6}$, Sofía González-Ortiz, Albert Lladó ${ }^{2,4}$, Rafael Blesa ${ }^{1,2}$, Bradford C. Dickerson ${ }^{8,9}$, Howard J. Rosen $^{5}$, Bruce L. Miller ${ }^{5}$, Alberto Lleó 1,2, Maria Luisa Gorno-Tempini ${ }^{5}$, Raquel Sánchez-Valle ${ }^{2,4}$ and Juan Fortea ${ }^{1,2,10^{*}}$

\begin{abstract}
Background Cortical mean diffusivity is a novel imaging metric sensitive to early changes in neurodegenerative syndromes. Higher cortical mean diffusivity values reflect microstructural disorganization and have been proposed as a sensitive biomarker that might antedate macroscopic cortical changes. We aimed to test the hypothesis that cortical mean diffusivity is more sensitive than cortical thickness to detect cortical changes in primary progressive aphasia (PPA).

Methods In this multicenter, case-control study, we recruited 120 patients with PPA (52 non-fluent, 31 semantic, and 32 logopenic variants; and 5 GRN-related PPA) as well as 89 controls from three centers. The 3-Tesla MRI protocol included structural and diffusion-weighted sequences. Disease severity was assessed with the Clinical Dementia Rating scale. Cortical thickness and cortical mean diffusivity were computed using a surface-based approach.
\end{abstract}

Results The comparison between each PPA variant and controls revealed cortical mean diffusivity increases and cortical thinning in overlapping regions, reflecting the canonical loci of neurodegeneration of each variant. Importantly, cortical mean diffusivity increases also expanded to other PPA-related areas and correlated with disease severity in all PPA groups. Cortical mean diffusivity was also increased in patients with very mild PPA when only minimal cortical thinning was observed and showed a good correlation with measures of disease severity.

Conclusions Cortical mean diffusivity shows promise as a sensitive biomarker for the study of the neurodegeneration-related microstructural changes in PPA.

Keywords Diffusion, Magnetic resonance, Primary progressive aphasia, Alzheimer's disease, Frontotemporal lobar degeneration

${ }^{\dagger}$ Ignacio Illán-Gala and Victor Montal contributed equally to this work.

*Correspondence:

Ignacio Illán-Gala

iillan@santpau.cat

Juan Fortea

jfortea@santpau.cat

Full list of author information is available at the end of the article

\section{Introduction}

Primary progressive aphasia (PPA) encompasses different neurodegenerative syndromes characterized by prominent deterioration of speech and language, and relative sparing of other cognitive functions [1, 2]. In 2011, an international group of experts introduced a common framework in which three variants of PPA were recognized, based on specific speech and language features: otherwise in a credit line to the material. If material is not included in the article's Creative Commons licence and your intended use is not permitted by statutory regulation or exceeds the permitted use, you will need to obtain permission directly from the copyright holder. To view a copy of this licence, visit http://creativecommons.org/licenses/by/4.0/. The Creative Commons Public Domain Dedication waiver (http://creativecommons.org/publicdomain/zero/1.0/) applies to the data made available in this article, unless otherwise stated in a credit line to the data. 
the non-fluent agrammatic variant (nfvPPA), the semantic variant (svPPA), and the logopenic (lvPPA) variant [3].

Currently, there are no disease-modifying treatments for PPA, but many pharmacological and non-pharmacological treatments are being developed with promising results [4]. However, several factors hamper the design of clinical trials in PPA. First, PPA cases are relatively rare, limiting the recruitment of participants [5]. Second, the diagnosis of PPA is difficult at very mild stages, when disease-modifying interventions are more likely to be effective, but atrophy can be subtle [6]. Finally, the prognosis of each PPA variant is heterogeneous and the progression rate can vary substantially within a given PPA variant $[7$, 8]. In this complex scenario, biomarkers represent powerful tools for the design of clinical trials [7]. Particularly, future clinical trials would largely benefit from instruments sensitive to the earliest neurodegenerative changes to increase diagnostic certainty, and optimize the measurement of treatment effects $[7,8]$.

Quantitative analysis of brain structure with magnetic resonance imaging (MRI) can detect disease-specific abnormalities and enable the comparison with normal populations and the determination of the vulnerable regions in each syndrome. Previous MRI studies have shown that each PPA variant is characterized by a relatively focal onset, and spreading along highly connected cerebral regions [9-12]. To date, most neuroimaging studies in PPA have focused on the cortical macrostructure with different metrics (gray matter density in voxelbased morphometry studies or cortical thickness in surface-based analyses) or white matter microstructural properties (namely diffusion tensor imaging metrics such as fractional anisotropy). However, diffusion tensor imaging can also be used to measure the magnitude of diffusivity (mean diffusivity), in the cerebral cortex [13]. Higher cortical mean diffusivity values reflect microstructural disorganization and disruption of cellular membranes and have been proposed as a sensitive biomarker that might antedate macroscopic cortical changes $[13,14]$. We have previously shown that diffusion imaging can also be used to measure the structural organization of the cerebral cortex and that such changes may reveal neurodegeneration that is not detected with cortical thickness measurements in different neurodegenerative diseases [14-16], even in the preclinical phase [17, 18].

In this multicenter study, our objective was to compare the cortical thickness and cortical mean diffusivity changes in patients with PPA. We also aimed to correlate these changes with clinical measures of disease severity. We hypothesized that cortical mean diffusivity is more sensitive than cortical thickness to detect cortical changes in PPA.

\section{Materials and methods Study population}

Participants with PPA were recruited from two cohorts, at three different centers: 88 at the Memory and Aging Center (MAC) of University of California, San Francisco (UCSF, CA, USA), and 72 at the Catalan Frontotemporal Dementia Initiative (CATFI; 32 at Hospital de Sant Pau and 40 at the Hospital Clinic de Barcelona, Barcelona, Spain). In both cohorts, the diagnosis was made by neurologists with expertise in the evaluation of PPA $[19,20]$. All patients underwent a complete clinical history, physical examination, neuropsychological evaluation, genetic screening for major FTLD mutations (for additional details, please refer to the "Genetic studies, biomarkers, and pathological assessment" section) and structural brain imaging [15, 21, 22]. A total of 92 age-matched healthy controls from the two cohorts were also included as imaging controls (35 UCSF and 57 CATFI). All healthy controls had normal cognitive performance according to normative data and did not have any neurologic, psychiatric, or other major medical illnesses [20, 23].

Inclusion criteria for PPA participants were (i) meeting basic PPA criteria as defined by Mesulam [1], (ii) meeting international criteria for one of the three PPA variants [3], and (iii) 3T MRI study available for structural and cortical mean diffusivity analysis (see below for details). Participants fulfilling basic PPA criteria that were found to have a mutation in the GRN gene $(n=5)$ were classified in a separate group because these cases are characterized by a widespread and asymmetric pattern of gray matter loss $[24,25]$ and usually display clinical features of more than one PPA subtype [26]. Figure 1 shows a flowchart of the sample composition. A total of 252 participants with appropriate $3 \mathrm{~T}$ structural and diffusionweighted MRI were considered for analysis. Of these, 43 (17\%) participants were excluded due to quality control issues or processing errors.

\section{Measures of disease severity and global cognitive function} At presentation, the CDR Dementia Staging Instrument plus National Alzheimer's Coordinating Center Behavior and Language Domains sum of boxes $\left(\mathrm{CDR}^{\circledR}\right.$ plus NACC FTLD-SB) scores were recorded as a measure of global of disease severity $[27,28]$. The CDR ${ }^{\circledR}$ plus NACC FTLD-SB was designed to apply the $\mathrm{CDR}^{\circledR}$ in FTLD natural history studies. It includes cognitive/functional domains: memory, orientation, judgment and problem solving, community affairs, home and hobbies, personal care, behavior, and language. Each category domain is rated on a 5-point scale ranging from 0 (normal), 0.5 (questionably or minimally impaired), 1 (mildly but definitely impaired), 2 (moderately impaired), to 3 (severely impaired). All ratings are based on the semi-structured interview from 


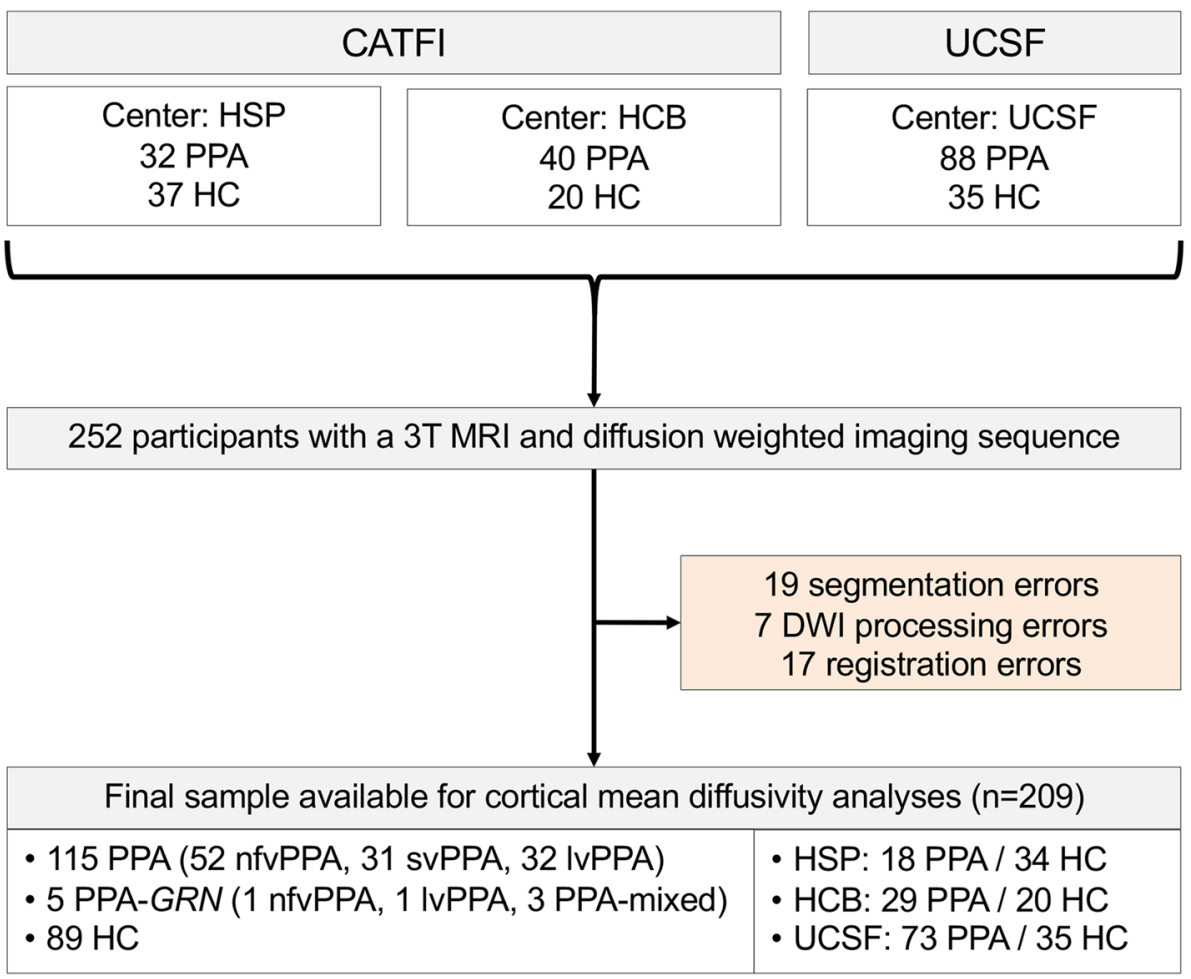

Fig. 1 Flowchart of the sample composition. Abbreviations: CATFI, Catalan Frontotemporal Dementia Initiative; FTLD, frontotemporal lobar degeneration; $\mathrm{HC}$, healthy controls; IVPPA, logopenic variant of primary progressive aphasia; MRI, magnetic resonance image; nfvPPA, non-fluent/ agrammatic variant of primary progressive aphasia; PPA, primary progressive aphasia; SVPPA, semantic variant of primary progressive aphasia; UCSF, University of California San Francisco

both a patient and a knowledgeable informant (typically a close family member of a patient) and do not consider neuropsychological assessments. The $\mathrm{CDR}^{\circledR}$ plus NACC FTLD-SB score (ranging from 0 to 24) is obtained by adding the rating of each domain. The global $\mathrm{CDR}^{\circledR}$ plus NACC FTLD (ranging from 0 to 3 ) score was also calculated following recently published scoring rules [28] to identify PPA participants at a mild stage of disease (global CDR ${ }^{\circledR}$ plus NACC FTLD score of 0.5). CDR ${ }^{\circledR}$ plus NACC FTLD-SB was available in 183 (88\%) of participants. The Mini-Mental State Examination (MMSE) was recorded in all centers as a general measure of cognitive impairment [29].

Genetic studies, biomarkers, and pathological assessment $A P O E$ was genotyped in all participants according to previously described methods [30]. Patients were screened for genetic mutations known to cause frontotemporal lobar degeneration and Alzheimer's disease (AD) [15, 21]. Genetic screening was conducted for mutations known to cause autosomal dominant FTLD or AD (MAPT, C9orf72, GRN, TARDBP, FUS, PSEN1, PSEN2, and APP) at each site, as previously described [15, 21].
Amyloid PET and core AD biomarkers were also available in a subset of participants. Amyloid PET studies were read as positive or negative, as previously described [20], and were performed with two different tracers ${ }^{11} \mathrm{C}$-Pittsburgh compound B at UCSF $[n=37]$ and with ${ }^{18}$ F-Florbetapir at CATFI $[n=4])$. The core AD biomarkers were available for CATFI participants and were performed as previously described [20,31].

Neuropathologic assessments performed at UCSF or Barcelona's Neurological Tissue Bank followed previously described procedures [20, 21]. Participants were classified into FTLD major molecular classes (tau, TDP-43, or FUS) and subtypes [22] or AD [32].

\section{MRI}

\section{MRI acquisition}

All participants underwent a 3T MRI using 4 different scanners. The acquisition parameters by the scanner can be found in Supplementary Table 1-2. All centers had a structural 3D MPRAGE T1-weighted acquisition of approximately $1 \times 1 \times 1 \mathrm{~mm}$ isotropic resolution and an EPI diffusion-weighted acquisition of at least $2.7 \times 2.7 \times$ $2.7 \mathrm{~mm}$ isotropic resolution. 


\section{Cortical thickness processing}

Cortical thickness reconstruction was performed with the Freesurfer package v6 ("FreeSurfer," n.d.) using a previously described procedure [33]. All individual cortical reconstructions were visually inspected on a slice-byslice basis to check for accuracy of the gray/white matter boundary segmentation. From the initial 252 participants with 3T MRI available from the three centers, 19 were excluded due to segmentation issues. Finally, each individual reconstructed brain was registered, and cortical thickness maps were morphed, to the fsaverage standard surface provided by Freesurfer, using a spherical registration, enabling an accurate inter-subject matching of cortical locations for the computation of further statistics. Before statistical analyses, we smoothed the cortical thickness maps using a Gaussian kernel with FWHM of $15 \mathrm{~mm}$ as implemented in Freesurfer [34].

\section{Cortical mean diffusivity processing}

We used a previously described home-made surfacebased approach to process cortical diffusion MRI [14]. Recent studies have shown the potential of surface-based methods to measure microstructural changes in neurodegenerative diseases [35] and the cortical architecture [36]. An important advantage of these methods is the mitigation of partial volume effects or kernel-sensitive CSF signal inclusion during the smoothing step [37]. Briefly, diffusion-weighted imaging data were first corrected for motion effects applying a rigid body transformation between the $b=0$ image and the diffusion-weighted acquisitions. Then, after removing non-brain tissue using the Brain Extraction Tool, diffusion tensors were fitted, and mean diffusivity was computed using the FSL's dtifit command. We then computed the affine transformation between the skull-stripped b0 and the segmented T1-weighted volume using a boundary-based algorithm as implemented in Freesurfer's bbregister. This approach takes advantage of the accurate segmentation of the white matter surface and pial surface obtained during Freesurfer's segmentation (cortical thickness processing section), to accurately register the b0 and the T1-weighted image, maximizing the intensity gradient across gray matter and white matter between both volumes. At this point, all the diffusion to $\mathrm{T} 1$ registrations were visually inspected to exclude those subjects with an erroneous co-registration. Seven participants were excluded due to diffusionweighted imaging processing errors. Then, the mean diffusivity volume for each individual was sampled at the midpoint of the cortical ribbon (half the distance along the normal vector between the white matter surface and the gray matter surface) and projected to each surface reconstruction obtained during the Freesurfer processing, to create a surface map of cortical mean diffusivity (using Freesurfer's mri_vol2surf command). Finally, individual cortical mean diffusivity maps were normalized to an average standard surface using a spherical registration, enabling an accurate inter-subject matching of cortical locations for the statistical analyses. Before statistical analyses, we applied a Gaussian kernel of $15 \mathrm{~mm}$ as implemented in Freesurfer [34], to obtain equivalent data effective smoothing between cortical thickness and cortical mean diffusivity.

\section{Cortical mean diffusivity harmonization between centers}

Because diffusion tensor imaging metrics are very sensitive to acquisition parameters, harmonization approaches are required to mitigate center-specific differences in multicenter studies. We applied a multicenter harmonization algorithm based on ComBat, to reduce center-specific differences in cortical mean diffusivity quantifications before any statistical analysis [38]. Briefly, ComBat uses an empirical Bayes framework to estimate the additive (mean) and multiplicative (variance) contribution of each site, at each vertex, for a specific diffusion tensor imaging metric and corrects these effects. Importantly, this approach allows the inclusion of biological information (such as clinical group or age), and it has been reported to preserve within-site biological variability, thereby increasing the statistical power [38].

\section{Statistical methods}

Data were explored for normality using the Shapiro-Wilk test. When necessary, variables were log-transformed using the natural $\log$ to fulfill the normal distribution assumption. Between-group differences were determined with ANOVA or $t$-test for continuous variables (with Bonferroni correction for multiple comparisons) and the chi-square for dichotomous or categorical data. We first performed group comparisons for cortical thickness and cortical mean diffusivity with a two-class general linear model, as implemented in Freesurfer, comparing each PPA variant to the control group. These analyses were repeated for each center independently. Moreover, we also performed additional analyses including only PPA participants with a global CDR ${ }^{\circledR}$ plus NACC FTLD score of 0.5 (mild PPA). Next, we performed a vertex-wise correlation analysis in each PPA variant group between the cortical thickness and cortical mean diffusivity and the $\mathrm{CDR}^{\circledR}$ plus NACC FTLD-SB (as a general measure of disease severity) and the MMSE (as a general measure of cognitive function). Specifically, a general linear model was created in which cortical mean diffusivity or cortical thickness was included as the dependent variable, and $\mathrm{CDR}^{\circledR}$ plus NACC FTLD-SB scores (and MMSE scores) were independent variables. We included age, sex, handedness, and center as nuisance variables in the cortical 
thickness analysis. In mean diffusivity analysis, only age, sex, and handedness were included since diffusion tensor imaging data were already harmonized between centers in a previous step. Only results that survived multiple comparisons (family-wise error $<0.05$ ) based on Monte Carlo simulation with 10,000 repeats as implemented in Freesurfer are presented. We used a very stringent threshold of $\alpha=0.001$ for the group analyses including all PPA participants and $\mathrm{HC}$, and a threshold of $\alpha=$ 0.05 for the analyses including PPA subgroups and the correlation analyses. A full description of the multiple comparison's methodology can be found in the Supplementary material.

We computed the Cohen's $d$ effect size metric for both cortical thickness and cortical mean diffusivity, on a vertex-wise basis, to obtain a topographical representation of the effect size for the group comparison between participants with PPA and controls. Effect size computation was restricted to cortical regions showing statistically significant differences between participants with PPA and controls for either cortical thickness or cortical mean diffusivity. We then computed the difference between the cortical thickness and cortical mean diffusivity effect size maps to obtain a topographical representation of the net effect size for each metric.

\section{Results}

Table 1 shows the demographics and clinical features of the participants in the study. Age at MRI, years of education, and disease duration at MRI were similar between participants with PPA and controls and between PPA subgroups. Sex and handedness distribution were also similar between all groups. As expected, all PPA variants showed lower values of MMSE and $\mathrm{CDR}^{\circledR}$ plus NACC FTLD-SB scores compared to the control group. Participants in the lvPPA group had lower MMSE scores when compared to participants in the nfvPPA and PPA-GRN groups. APOE 44 carriers were overrepresented in the lvPPA group (33\%) compared to the nfvPPA, svPPA, and GRN-PPA groups $(19 \%, 16 \%$, and $0 \%$, respectively). The

Table 1 Characteristics of the participants

\begin{tabular}{|c|c|c|c|c|c|c|}
\hline & \multicolumn{3}{|l|}{ PPA } & \multirow[b]{2}{*}{$\begin{array}{l}\text { PPA-GRN } \\
n=5(4 \%)\end{array}$} & \multirow[b]{2}{*}{$\begin{array}{l}\text { All PPA } \\
n=120(100 \%)\end{array}$} & \multirow{2}{*}{$\begin{array}{l}\mathrm{HC} \\
\mathrm{HC} \\
n=89\end{array}$} \\
\hline & $\begin{array}{l}\text { nfvPPA } \\
n=52(43 \%)\end{array}$ & $\begin{array}{l}\text { svPPA } \\
n=31(26 \%)\end{array}$ & $\begin{array}{l}\text { IvPPA } \\
n=32(27 \%)\end{array}$ & & & \\
\hline Cohort, no. (CATFI/UCSF) & $18 / 34$ & $15 / 16$ & $11 / 21$ & $3 / 2$ & $47 / 73+$ & $54 / 35+$ \\
\hline \multicolumn{7}{|l|}{ Demographics, genetic, and clinical features } \\
\hline Age at MRl, years & $69.0(7.3)$ & $67.1(7.5)$ & $66.3(7.9)$ & $61.7(3.3)$ & $67.5(7.5)$ & $66.0(6.9)$ \\
\hline Biological sex (men/women) & $20 / 32$ & $16 / 15$ & $16 / 16$ & $0 / 5$ & $52 / 68$ & $31 / 58$ \\
\hline APOE $\varepsilon 4$, no. (positive/negative) & $9 / 39$ & $4 / 21$ & $9 / 18$ & $0 / 4$ & $22 / 82$ & $7 / 28$ \\
\hline Education, years & $14.2(5.2)$ & $14.6(4.0)$ & $13.1(7.2)$ & $12.4(5.7)$ & $13.9(5.5)$ & $14.7(4.4)$ \\
\hline Handedness (right/left) & $45 / 7$ & $28 / 3$ & $29 / 3$ & $4 / 1$ & $106 / 14$ & $85 / 4$ \\
\hline Disease duration at MRI, years & $4.2(2.3)$ & $4.5(3.2)$ & $4.1(1.8)$ & $2.5(1.6)$ & $4.2(2.4)$ & - \\
\hline \multicolumn{7}{|l|}{ Measures of cognition and disease severity } \\
\hline MMSE, /30 & $25.0(4.6) \neq$ & $23.9(4.6)$ & $20.7(6.2) \neq$ & $27.0(2.6) \neq$ & $23.7(5.3) \dagger$ & $29.1(0.9) \dagger$ \\
\hline $\mathrm{CDR}^{\circledR}$ plus NACC FTLD-SB, $/ 24^{\mathrm{a}}$ & $3.9(2.6) \S$ & $6.3(3.2) \S$ & $5.1(2.8)$ & $3.8(4.6)$ & $4.9(3.0)+$ & $0.0(0) \dagger$ \\
\hline Global CDR ${ }^{\circledR}$ plus NACC FTLD $=0.5$, no. $(\%)$ & $20(38)$ & $6(19)$ & $9(28)$ & $1(20)$ & $36(30) \dagger$ & $0(0) \dagger$ \\
\hline \multicolumn{7}{|l|}{ Biomarkers and autopsy findings } \\
\hline Amyloid PET, no. (positive/negative) & $0 / 21$ & $1 / 6$ & $13 / 0$ & $0 / 0$ & $14 / 27$ & $1 / 1$ \\
\hline AD biomarkers in CSF, no. (Positive/Negative) ${ }^{b}$ & $3 / 12$ & $2 / 12$ & $9 / 0$ & $0 / 2$ & $14 / 26$ & $5 / 27$ \\
\hline Pathological diagnosis, no. $(F T L D / A D)^{c}$ & $16 / 0$ & $3 / 0$ & $0 / 8$ & $2 / 0$ & $21 / 8$ & $0 / 0$ \\
\hline
\end{tabular}

Abbreviations: AD, Alzheimer's disease; CATFI, Catalan Frontotemporal Dementia Initiative; CDR, Clinical Dementia Rating; CDR ${ }^{\circledR}$ plus NACCFTLD-SB, CDR Dementia Staging Instrument plus National Alzheimer's Coordinating Center Behavior and Language Domains sum of boxes; MRI, magnetic resonance image; MMSE, MiniMental State Examination; UCSF, University of California San Francisco; IVPPA, logopenic variant of primary progressive aphasia; $n f v P P A$, non-fluent/agrammatic variant of primary progressive aphasia; SVPPA, semantic variant of primary progressive aphasia

${ }^{\text {a }}$ CDR $^{\circledR}$ plus NACC FTLD-SB was available in 183 (88\%) of participants (94 PPA and $89 \mathrm{HC}$ )

${ }^{b} A$ positive Alzheimer's disease biomarker profile was defined by an abnormal $A ß 1-42$ to $A ß 1-40$ ratio and a total-tau to $A ß 1-42$ ratio

${ }^{\mathrm{C}}$ The 21 FTLD cases included 6 cases with corticobasal degeneration (all in the nfvPPA group), 5 cases with progressive supranuclear palsy (all in the nfvPPA group), 5 cases with Pick's disease ( 4 in the nfvPPA group and 1 in the svPPA group), 2 cases with FTLD-TDP type A (all in the GRN-PPA group), 1 case with FTLD-TDP type B (in the svPPA group), 1 case with FTLD-TDP type $C$ (in the SVPPA group) and 1 case with an unclassifiable tauopathy (in the nfvPPA group)

tDifferent between all PPA group and $\mathrm{HC}$ group $(P<.05)$

‡Different between IvPPA, nfvPPA, and PPA-GRN ( $P<.05$, Bonferroni adjusted)

$\S$ Different between svPPA and nfvPPA $(P<.05$, Bonferroni adjusted) 
$\mathrm{CDR}^{\circledR}$ plus NACC FTLD-SB scores were higher in the svPPA group when compared to the nfvPPA group. The frequency of PPA participants with a global CDR ${ }^{\circledR}$ plus NACC FTLD score of 0.5 ranged from $19 \%$ in svPPA to $38 \%$ in nfvPPA.

As shown in Table 1, 29 (14\%) of the participants with PPA underwent autopsy evaluation. All the participants with a neuropathological diagnosis of Alzheimer's disease $(n=8)$ had a diagnosis of lvPPA. Conversely, all participants with a diagnosis of FTLD were classified in the nfvPPA $(n=16)$, svPPA $(n=3)$, and GRN-PPA $(n=2)$ groups.

We first compared cortical thickness and cortical mean diffusivity between PPA and controls. As shown in Fig. 2, the nfvPPA group showed the expected pattern of cortical thinning in the precentral, inferior, middle, and superior frontal gyri as well as supplementary motor and dorsomedial prefrontal cortex in both hemispheres, but higher effect sizes were observed in the left hemisphere. In this group, the cortical mean diffusivity map involved more regions, including the frontal pole, the insula, dorsal anterior and pregenual cingulate cortex, posterior cingulate and precuneus, and the temporo-parietal junction of both hemispheres as well as a large swatch of the left lateral temporal cortex (Fig. 2B). The svPPA showed the expected pattern of cortical thinning involving the lateral, ventral, and medial anterior temporal cortex and temporal pole, insula, subgenual anterior cingulate cortex, inferior parietal lobule, and caudal inferior frontal gyrus of the left hemisphere and, to a lesser extent, the anterior temporal cortex and insula of the right hemisphere (Fig. 2A). Cortical mean diffusivity changes were present in the same regions as thickness changes but extended beyond those to include the pregenual and dorsal anterior cingulate cortex of both hemispheres and the dorsolateral prefrontal cortex and the inferior parietal lobule of the left hemisphere (Fig. 2B). Participants within the lvPPA group displayed cortical thinning in the expected lateral temporal and inferior parietal lobules of both hemispheres, with additional thinning in superior

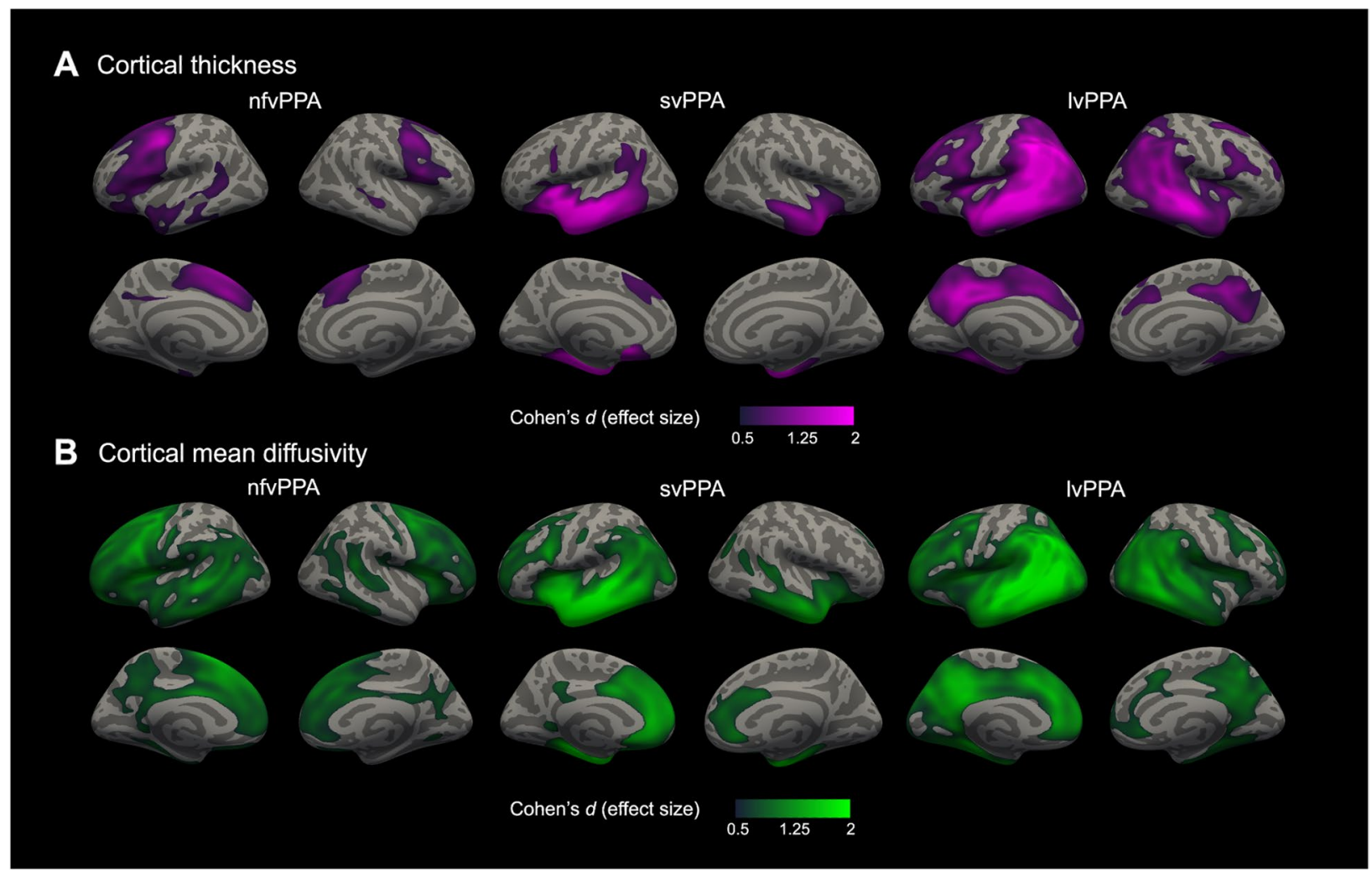

Fig. 2 Group comparison of cortical thickness and cortical mean diffusivity between participants with PPA and healthy controls. Statistically significant results between major PPA subtypes (52 nfvPPA, 31 svPPA, 32 IvPPA) and healthy controls $(n=89)$ for cortical thickness (A) and cortical mean diffusivity (B). Cortical thickness analyses were adjusted for age, sex, handedness, and MRI scan. Cortical mean diffusivity analyses were adjusted for age, sex, and handedness after a harmonization step. Only effect sizes (Cohen's $d$ ) inside clusters that survived family-wise error correction $(P<.001)$ are shown. Abbreviations: IVPPA, logopenic variant of primary progressive aphasia; nfvPPA, non-fluent/agrammatic variant of primary progressive aphasia; svPPA, semantic variant of primary progressive aphasia 
parietal lobules, prefrontal cortex, and posterior cingulate and precuneus cortex, with higher effect sizes in the left hemisphere (Fig. 2A). Again, in the lvPPA variant, we also observed more widespread cortical mean diffusivity increases involving those same regions as well as the medial prefrontal cortex and pregenual and dorsal anterior cingulate cortex of both hemispheres (Fig. 2B).

Thus, while cortical thickness and cortical mean diffusivity maps showed a partial overlap in all PPA variants, cortical mean diffusivity changes with at least moderate effect sizes extended beyond the areas of cortical thinning (Fig. 3). Importantly, the effect sizes of cortical mean diffusivity in overlapping areas were higher than the effect sizes of cortical thickness with the sole exception of neurodegeneration cores for each PPA variant (namely, the left temporal pole for svPPA, premotor cortex for nfvPPA, and temporo-parietal cortex for lvPPA). Moreover, we observed essentially the same patterns of cortical thickness and cortical mean diffusivity changes when each cohort was analyzed separately (data not shown).

Figure 4 shows cortical thickness and cortical mean diffusivity comparison between PPA participants with GRN mutation and controls. As expected, we observed cortical thinning in the prefrontal, lateral temporal, and inferior parietal cortex. In this group, cortical thinning was restricted to the left hemisphere. In the PPA-GRN group, we also observed widespread increases in cortical mean diffusivity affecting both hemispheres (Fig. 4).

Next, we explored group differences in cortical thickness and cortical mean diffusivity in the subgroup of participants with early-stage PPA (global $\mathrm{CDR}^{\circledR}$ plus NACC FTLD score of 0.5). As expected, we observed the same pattern of cortical thinning and cortical mean diffusivity increases. However, these changes were more restricted to the neurodegeneration cores of each PPA variant (Fig. 5).

Finally, we evaluated the capacity of cortical thickness and cortical mean diffusivity to reflect disease severity in PPA as measured by the CDR ${ }^{\circledR}$ plus NACC FTLD-SB scores. As shown in Fig. 6, we observed an inverse correlation between $\mathrm{CDR}^{\circledR}$ plus NACC FTLD-SB scores and cortical thickness in left-lateralized prefrontal regions in nfvPPA, widespread regions of the right frontal and temporal cortex in SVPPA, and no localizable effects in lvPPA. As with our other effects, we observed larger clusters with similar localization between cortical mean diffusivity and CDR ${ }^{\circledR}$ plus NACC FTLD-SB scores (Fig. 6). We observed similar results when studying the correlation of cortical thickness and cortical mean diffusivity with MMSE (Supplementary Fig. 1).

\section{Discussion}

This large multicenter study investigated cortical mean diffusivity in PPA. Importantly, we showed that cortical mean diffusivity increases not only coincided with areas that showed cortical thinning but also involved other areas that typically become affected later during disease progression. Cortical mean diffusivity was increased in patients with very mild PPA when only minimal cortical thinning was observed. Finally, we also explored the

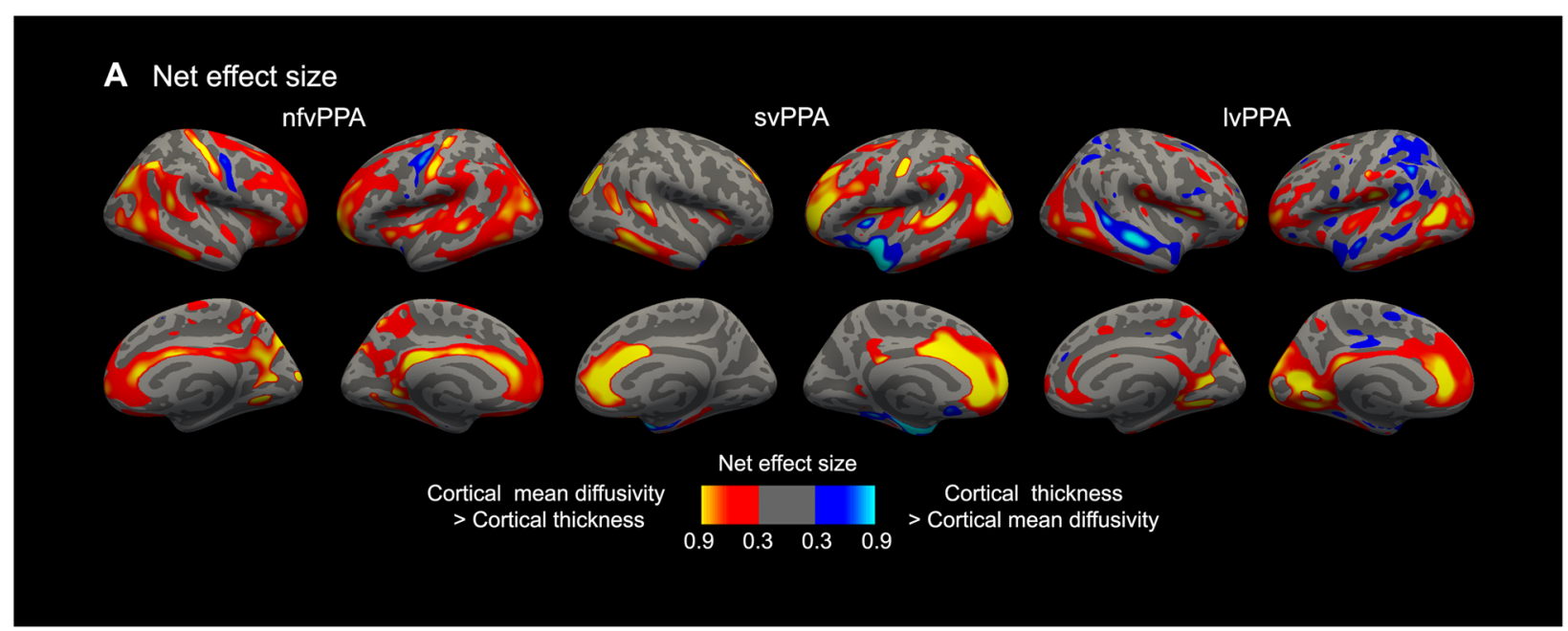

Fig. 3 Net effect size. The net effect size (A) was obtained by subtracting the cortical thickness and cortical mean diffusivity effect size maps of the comparison between PPA participants and healthy controls. The red-yellow color represents cortical areas where the cortical mean diffusivity has a higher effect size than cortical thickness. The blue color represents cortical areas where the cortical thickness has a higher effect size than cortical mean diffusivity. Abbreviations: IvPPA, logopenic variant of primary progressive aphasia; nfvPPA, non-fluent/agrammatic variant of primary progressive aphasia; svPPA, semantic variant of primary progressive aphasia 


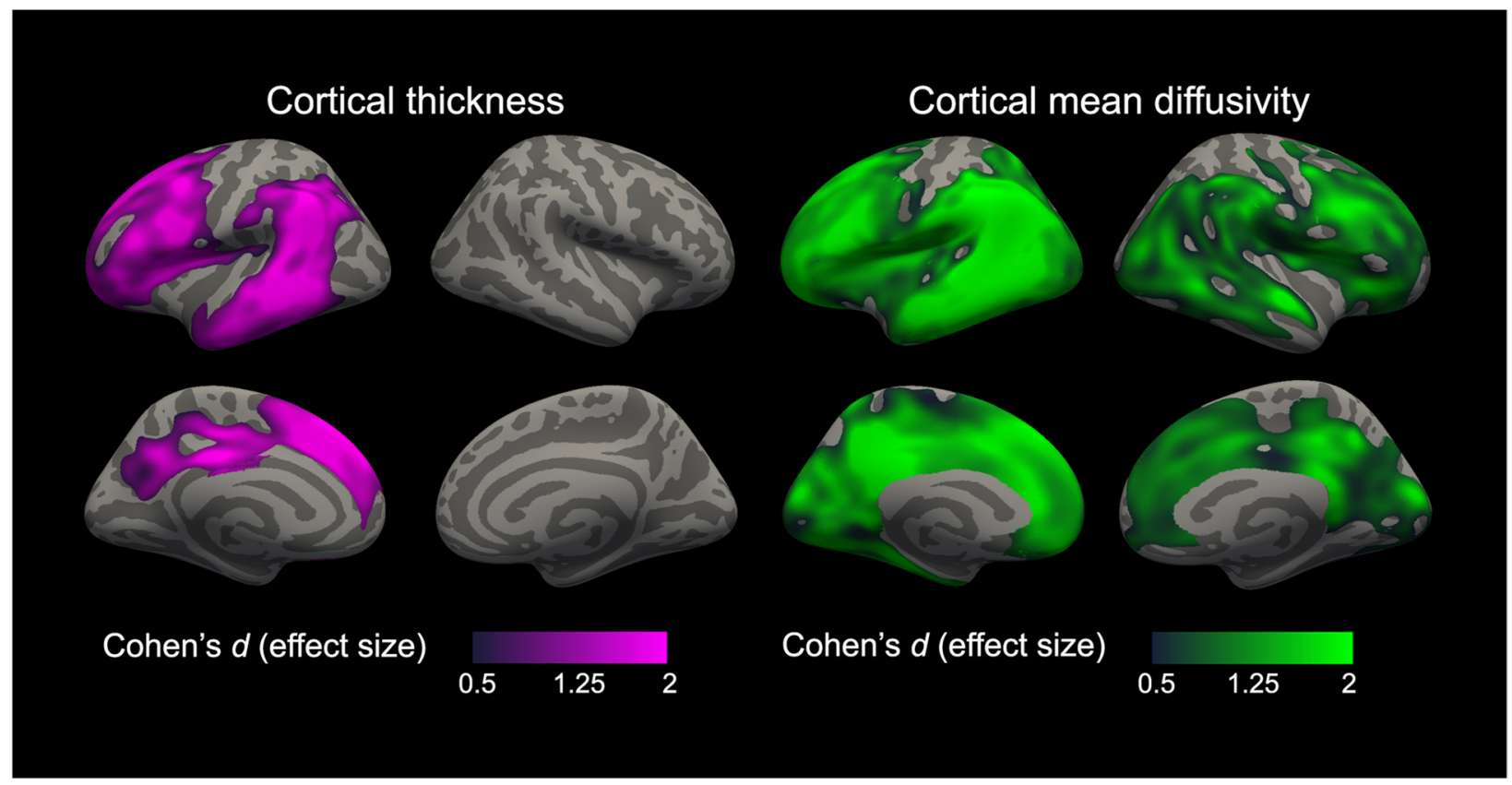

Fig. 4 Group comparison of cortical thickness and cortical mean diffusivity between participants with GRN mutation and healthy controls. Statistically significant results between PPA participants with GRN mutation $(n=5)$ and healthy controls for cortical thickness (left) and cortical mean diffusivity (right). For these analyses, we only considered healthy controls scanned at the same MRI than PPA participants with GRN mutation $(n=38)$. Cortical thickness analyses were adjusted for age, sex, handedness, and MRI scan. Cortical mean diffusivity analyses were adjusted for age, sex, and handedness after a harmonization step. Only effect sizes (Cohen's $d$ ) inside clusters that survived family-wise error correction $(P<.05)$ are shown

correlation between cortical mean diffusivity and clinical measures of disease severity and general cognitive function. Taken together, these findings suggest that cortical mean diffusivity might be more sensitive than cortical thickness to detect the earliest disease-related cortical changes in PPA.

Cortical mean diffusivity has been recently proposed as a sensitive biomarker for the detection of the earliest cortical changes in sporadic Alzheimer's disease and the Amyotrophic Lateral Sclerosis-Frontotemporal dementia continuum [13, 14, 16-18]. This study expands these findings to PPA. We showed that cortical mean diffusivity increases spread beyond the areas of cortical thinning in PPA. Most previous studies using diffusion tensor imaging in PPA patients have focused on the white matter, probably because of the technical difficulties in the study of cortical microstructure. One study found overlapping patterns between atrophy and increases in cortical mean diffusivity in the nfvPPA and semantic dementia [39]. Our study builds on these results using a larger sample, a surface-based approach, and the inclusion of all PPA variants included in the 2011 consensus criteria. Consequently, we were able to explore the added value of cortical mean diffusivity over cortical thickness, particularly in mildly symptomatic cases.

PPA variants are characterized by a focal onset of neurodegeneration spreading along specific networks [40]. We observed cortical thinning including the core of neurodegeneration of each PPA when comparing cortical thickness and cortical mean diffusivity of PPA participants and controls. Importantly, we also found cortical mean diffusivity increases beyond the regions with atrophy in regions that are known to become involved with disease progression. The effect size maps showed moderate to high net effect size favoring cortical mean diffusivity in key regions for each PPA subtype except in the neurodegenerative core of each syndrome. Although the origin of these microstructural changes is unclear, higher cortical mean diffusivity values have been found to reflect a loss of tissue integrity and breakdown of cell membranes in the cortex. We also hypothesize that cortical mean diffusivity might reach floor effects earlier than atrophy. Hence, the very focal atrophy seen at the epicenters in PPA (particularly in the svPPA variant) [11] is better reflected with cortical thickness, but the spread of pathology to other cortical areas may be better captured by cortical mean diffusivity. 


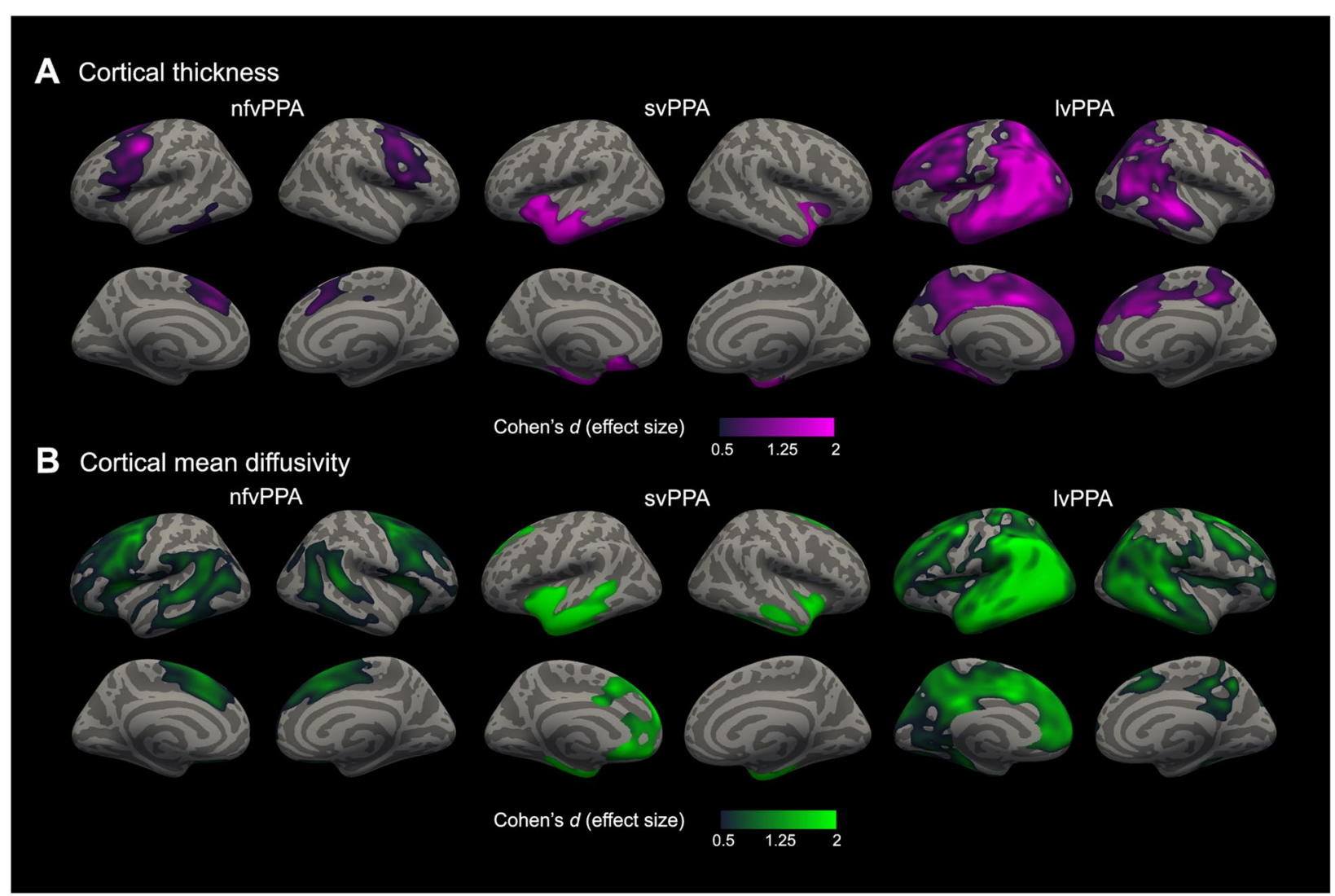

Fig. 5 Group comparison of cortical thickness and cortical mean diffusivity between participants with mild PPA and healthy controls. Statistically significant results between participants with mild PPA (as defined by a global CDR ${ }^{\circledR}$ plus NACC FTLD score of 0.5; 20 nfvPPA, 6 svPPA, and 9 lvPPA) and healthy controls for cortical thickness (A) and cortical mean diffusivity (B). Cortical thickness analyses were adjusted for age, sex, handedness, and MRI scan. Cortical mean diffusivity analyses were adjusted for age, sex, and handedness after a harmonization step. Only effect sizes (Cohen's $d$ ) inside clusters that survived family-wise error correction $(P<.001)$ are shown. Abbreviations: IvPPA, logopenic variant of primary progressive aphasia; nfvPPA, non-fluent/agrammatic variant of primary progressive aphasia; sVPPA, semantic variant of primary progressive aphasia

Furthermore, we performed an exploratory analysis in the PPA participants with GRN mutations. We classified PPA participants with GRN mutations in a separate group because these cases are characterized by a more widespread and asymmetric pattern of gray matter loss. In the PPA-GRN group, cortical thinning was restricted to the left hemisphere, but cortical mean diffusivity extended to both hemispheres. This finding also supports the view that cortical mean diffusivity is capturing early neurodegenerative changes antedating overt neuronal loss and cortical thinning. This observation in the PPAGRN group also encourages the investigation of cortical mean diffusivity in larger samples of FTLD mutation carriers.

The suggestion that cortical mean diffusivity may be more sensitive than cortical thickness to detect the PPA cortical changes is further supported by our correlation analyses with the $\mathrm{CDR}^{\circledR}$ plus NACC FTLD-SB and MMSE scores. The $\mathrm{CDR}^{\circledR}$ plus NACC FTLD-SB has been validated as a tool for disease monitoring in clinical trials $[28,41]$. Although the $\mathrm{CDR}^{\circledR}$ plus NACC FTLD-SB scores correlated with cortical thickness in some small frontotemporal clusters, we found a stronger and more widespread correlation with cortical mean diffusivity. These results should be, however, considered exploratory. More studies are needed to determine the precise relationship between cortical mean diffusivity and FTLDrelated neurodegeneration, from the preclinical to the dementia stage.

Although clinical-pathological correlations are not perfect, each PPA variant is associated with typical pathologies. For instance, in the absence of a GRN mutation, the nfvPPA syndrome is frequently associated with Tau subtypes of FTLD, while the svPPA is associated with the TDP-C subtype of FTLD, and lvPPA is typically associated with AD pathologic changes [42]. We observed similar cortical mean diffusivity increases for each PPA group, suggesting that cortical mean diffusivity changes may be 


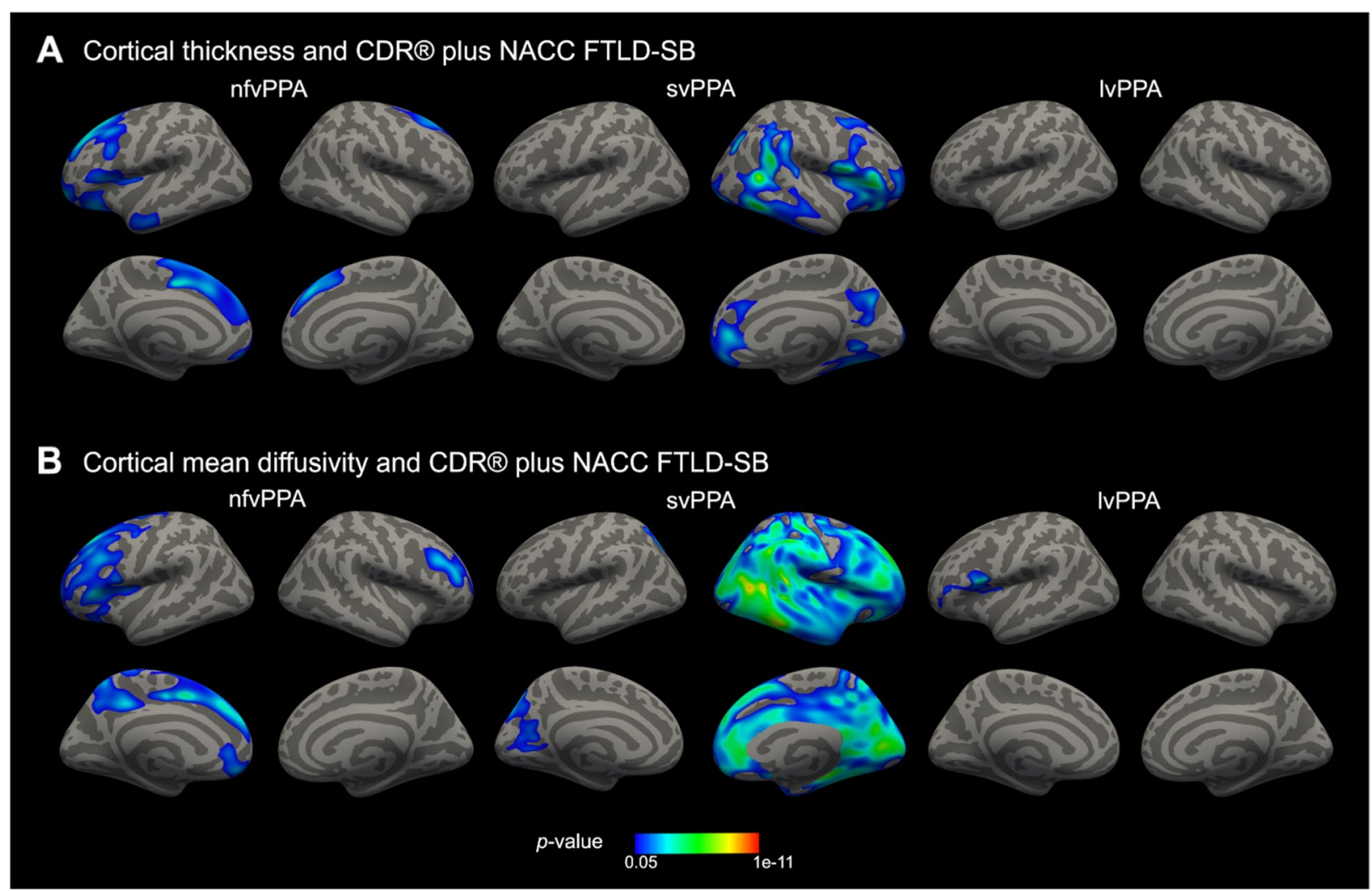

Fig. 6 Correlation of cortical thickness and cortical mean diffusivity with the CDR ${ }^{\circledR}$ plus NACC FTLD-SB. Relationship of cortical thickness (A) and cortical mean diffusivity (B) with the CDR ${ }^{\circledR}$ plus NACC FTLD scores. The CDR ${ }^{\circledR}$ plus NACC FTLD-SB scores were negatively correlated with cortical thickness and positively correlated with cortical mean diffusivity. Cortical thickness analyses were adjusted for age, sex, handedness, and MRI scan. Mean diffusivity analyses were adjusted for age, sex, and handedness after a harmonization step. Only clusters that survived family-wise error correction $(P<.05)$ are shown. Abbreviations: CDR, clinical dementia rating; $C D R^{\circledR}$ plus NACC FTLD-SB, CDR Dementia Staging Instrument plus National Alzheimer's Coordinating Center Behavior and Language Domains sum of boxes; IVPPA, logopenic variant of primary progressive aphasia; nfvPPA, non-fluent/agrammatic variant of primary progressive aphasia; sVPPA, semantic variant of primary progressive aphasia

an unspecific biomarker of neurodegeneration. However, the difference between cortical thickness and cortical mean diffusivity changes was less clear in the lvPPA group. In addition, the correlation between cortical mean diffusivity and disease severity was also less evident in the lvPPA group than in the nfvPPA and svPPA groups. Two important factors should be considered when interpreting these findings. First, the pattern of neurodegeneration in the lvPPA variant is more widespread and more heterogeneous than the pattern observed in other PPA variants, as noted by previous studies [43]. Importantly, a higher heterogeneity within a neurodegenerative syndrome penalizes finding statistically significant correlations between general measures of disease severity and neuroimage measures [15]. Second, the lvPPA syndrome is strongly associated with Alzheimer's disease, a disease characterized by the extraneuronal deposition of fibrillary amyloid, together with intraneuronal aggregation of tau. Of note, we have previously shown that amyloid deposition impacts cortical mean diffusivity, even in the preclinical phase of Alzheimer's disease [44]. Because only tau pathology (and not amyloid deposition) is closely correlated with neurodegeneration in Alzheimer's disease, we hypothesize that amyloid-related microstructural changes could attenuate the association between disease severity and cortical mean diffusivity in the lvPPA group [45]. However, additional multimodal imaging studies are needed to confirm this hypothesis and precise the relationship between amyloid deposition, tau pathology, and cortical microstructure in Alzheimer's disease. In addition, autopsy-proven studies should explore the added value of cortical mean diffusivity (alone or combined with white matter diffusion tensor imaging changes) to detect specific neuropathological signatures (i.e., prominent microstructural changes in the subcortical white matter in FTLD-Tau or widespread gray matter changes 
in Alzheimer's disease with relative sparing of subcortical white matter) [46-48].

Our findings support the role of cortical mean diffusivity as a potential neurodegeneration biomarker in PPA to be used in clinical trials. Trials of drugs targeting abnormal protein deposition need meaningful end points. Historically, these end points included neuropsychological scores and functional scales. Brain imaging could provide more sensitive and robust evidence of disease modification [49]. Our study shows that cortical mean diffusivity is a more sensitive biomarker than cortical thickness and correlates more strongly with clinical and cognitive measures, in agreement with a previous report [49]. The increased sensitivity of cortical mean diffusivity suggests it may be a sensitive tool suitable to monitor the earliest cortical changes in preclinical or mildly symptomatic phases of FTLD [50].

The main strengths of this study are the relatively large number of participants with PPA at a mild disease stage, and the surface-based analyses using a previously validated technique. This surface-based approach solves some of the limitations and methodological concerns that have been previously reported when using a voxel-based approach [37]. Moreover, we enriched our description of the cortical mean diffusivity in PPA and we were able to replicate our results in two different cohorts.

\section{Limitations}

This study has also some limitations. A proportion of participants with PPA were excluded due to segmentation or diffusion tensor imaging processing errors. Even though this is an inherent limitation of our surface-based approach, future improvements in T1 MRI acquisitions or the use of higher field MRIs will likely reduce the number of subjects excluded due to segmentation errors. Also, both the PPA-GRN group and the subgroup of PPA participants at a mild disease stage were small. Finally, further longitudinal studies in presymptomatic mutation carriers should confirm that cortical mean diffusivity changes track disease progression and antedate cortical atrophy in patients with PPA.

\section{Conclusions}

Cortical mean diffusivity shows promise as a sensitive biomarker for the study of the earliest neurodegeneration-related cortical changes in PPA. Further longitudinal studies including preclinical mutation carriers are needed to fully determine the potential utility of this biomarker to detect cortical changes antedating cerebral atrophy at the earliest stages of the disease and to track disease progression.

$\begin{array}{ll}\text { Abbreviations } & \\ \text { AD } & \text { Alzheimer's disease } \\ \text { CATFI } & \text { Catalan Frontotemporal Dementia Initiative } \\ \text { CDR } & \text { Clinical Dementia Rating } \\ \text { CDR } & \text { plus } \\ \text { NACC FTLD-SB } & \begin{array}{l}\text { CDR Dementia Staging Instrument plus National Alzheimer's } \\ \text { Coordinating Center Behavior and Language Domains sum } \\ \text { of boxes }\end{array} \\ \text { FDR } & \text { False discovery rate } \\ \text { FWE } & \text { Family-wise error } \\ \text { HC } & \text { Healthy control } \\ \text { MMSE } & \text { Mini-Mental State Examination } \\ \text { MRI } & \text { Magnetic resonance image } \\ \text { PPA } & \text { Primary progressive aphasia } \\ \text { UCSF } & \text { University of California San Francisco }\end{array}$

\section{Supplementary Information}

The online version contains supplementary material available at https://doi. org/10.1186/s13195-022-00974-0.

Additional file 1. Supplementary material.

\section{Acknowledgements}

The authors thank the patients and their relatives for their support for this study.

\section{Authors' contributions}

Ignacio Illan-Gala: drafting/revision of the manuscript for content, including medical writing for content; major role in the acquisition of data; study concept or design; analysis or interpretation of the data. Victor Montal: drafting/revision of the manuscript for content, including medical writing for content; analysis or interpretation of the data. Sergi Borrego-Écija: drafting/ revision of the manuscript for content, including medical writing for content; major role in the acquisition of the data. Maria Luisa Mandelli: drafting/revision of the manuscript for content, including medical writing for content; major role in the acquisition of the data; analysis or interpretation of the data. Neus Falgàs: drafting/revision of the manuscript for content, including medical writing for content. Ariane E. Welch: drafting/revision of the manuscript for content, including medical writing for content; major role in the acquisition of the data Jordi Pegueroles: drafting/revision of the manuscript for content, including medical writing for content Miguel Santos-Santos: drafting/revision of the manuscript for content, including medical writing for content; major role in the acquisition of the data. Alexandre Bejanin: drafting/revision of the manuscript for content, including medical writing for content. Daniel Alcolea: drafting/revision of the manuscript for content, including medical writing for content; major role in the acquisition of the data. Oriol Dols-lcardo: drafting/ revision of the manuscript for content, including medical writing for content. Olivia Belbin: drafting/revision of the manuscript for content, including medical writing for content. María Belén Sánchez-Saudinós: drafting/revision of the manuscript for content, including medical writing for content; major role in the acquisition of the data. Nuria Bargalló: drafting/revision of the manuscript for content, including medical writing for content; major role in the acquisition of the data. Sofía González-Ortiz: drafting/revision of the manuscript for content, including medical writing for content; major role in the acquisition of the data. Albert Llado: drafting/revision of the manuscript for content, including medical writing for content; major role in the acquisition of the data. Rafael Blesa: drafting/revision of the manuscript for content, including medical writing for content; major role in the acquisition of the data. Bradford C. Dickerson: drafting/revision of the manuscript for content, including medical writing for content; major role in the acquisition of the data. Howard J. Rosen: drafting/revision of the manuscript for content, including medical writing for content; major role in the acquisition of the data; analysis or interpretation of the data. Bruce L. Miller: drafting/revision of the manuscript for content, including medical writing for content; major role in the acquisition of the data. Alberto Lleó: drafting/revision of the manuscript for content, including medical writing for content; major role in the acquisition of the data. Maria Luisa Gorno-Tempini: drafting/revision of the manuscript for content, including medical writing for content; major role in the acquisition of the data; analysis 
or interpretation of the data. Raquel Sánchez-Valle: drafting/revision of the manuscript for content, including medical writing for content; major role in the acquisition of the data. Juan Fortea: drafting/revision of the manuscript for content, including medical writing for content; major role in the acquisition of the data; study concept or design; analysis or interpretation of the data. The authors read and approved the final manuscript.

\section{Authors' information}

Not applicable

\section{Funding}

I.I.G. and N.F. are Atlantic Fellow for Equity in Brain Health at the Global Brain Health Institute (GBHI), and I.I.G. is supported with funding from $\mathrm{GBHI}$, Alzheimer's Association, and Alzheimer's Society (GBHI ALZ UK-21-720973). I.I.G. is also supported by the Juan Rodés Contract (JR20/0018) from Instituto de Salud Carlos III. V.M. is supported by a PFIS contract (FI18/00275) from the Carlos III Health Institute. S.B.E. is the recipient of Emili Letang post-residency research grant from Hospital Clínic de Barcelona. A.E.W received salary from UCSF (supported by NIH grants). A.B. is supported by a Miguel Servet I grant (CP20/00038) from the Carlos III Health Institute and the postdoctoral Fellowship, "Juan de la Cierva Incorporación," from the Spanish Ministry of Science, Innovation, and Universities. O.D.I. is a recipient of a grant by The Association for Frontotemporal Degeneration (Clinical Research Postdoctoral Fellowship, AFTD 2019-2021) and FUNDELA. The Catalan Frontotemporal Initiative (CATFI) was funded by the Health Department of the Government of Catalonia (grant PERIS SLT002/16/00408 to A.Lleó and R.S.V). This work was supported by NIH grants AG019724, AG032306, AG045390, NS092089, AG045333, AG056749, AG062422, P30AG062422, and P01AG019724. This work was also supported by the National Institutes of Health (NIA grants 1R01AG056850-01A1; R21AG056974; and R01AG061566 to JF) and by "Fondo de Investigaciones Sanitario" (FIS), Instituto de Salud Carlos III (PI14/01126 and PI17/01019 to JF, PI13/01532 and PI16/01825 to RB, PI18/00335 to MCI, INT19/00016 and PI18/00435 to DA, PI15/01618 to R.R., PI20/00448 to R.S-V, and PI17/01896, PI20/01330 and AC19:00103 to A.Lleó), the CIBERNED program (Program 1, Alzheimer Disease to A.Lleó and SIGNAL study,www.signalstudy.es), partly jointly funded by Fondo Europeo de Desarrollo Regional, Unión Europea, una manera de hacer Europa, the "Marató TV3" grant (20141210 to J.F., 044412 to R.B., and 20143810 to R. S.V), by Generalitat de Catalunya (2014SGR-0235 to A.Lleó, PERIS SLT006/17/125 to D.A., and SLT006/17/00119 to J. F.), by BBVA foundation (grant to A. Lleó), by a grant from the Fundació Bancaria La Caixa to R.B. and by Sage Pharma to R.S-V (HCB2020/1483). European Union's Horizon 2020, 'MES-CoBraD' (H2020-SC1-BHC-2018-2020/GA 965422 to JF).

\section{Availability of data and materials}

The datasets analyzed during the current study are available from the corresponding authors on reasonable request.

\section{Declarations}

\section{Ethics approval and consent to participate}

Informed consent was obtained from all subjects, and local institutional review boards for human research approved the study.

\section{Consent for publication}

All the co-authors have participated actively in this work and have read and agreed to this final version of the manuscript.

\section{Competing interests}

I.I.G. reports no disclosures; V.M. reports no disclosures; S.B.E. reports no disclosures; M.L.M. reports no disclosures; N.F. reports no disclosures; A.E.W reports no disclosures; J.P. received an award from the Spanish Society of Neurology (best communication award); M.S.S reports no disclosures; A.B. was awarded in 2019 with the travel Fellowship from the Alzheimer's Association to attend to the Alzheimer's Association International Conference (AAIC); D.A. has a patent (WO2019175379 A1 Markers of synaptopathy in neurodegenerative disease) and participated in advisory boards from Fujirebio-Europe and Roche Diagnostics and speaker honoraria from Fujirebio-Europe, Roche Diagnostics, Nutricia, Krka Farmacéutica S.L., Zambon S.A.U., and Esteve Pharmaceuticals S.A; O.D.I reports no disclosures; O.B. has a patent (WO2019175379 A1 markers of synaptopathy in neurodegenerative disease); B.S.S. reports no disclosures;
N.B. is member of the executive committee of the European Society of Radiology and reports examiner honoraria from the European Board of Neuroradiology; S.G.O. reports no disclosures; A. Llado reports no disclosures; R.B. reports honoraria from Nutricia; B.C.D reports no disclosures; H.J.R. reports honoraria from Biogen, Wave neuroscience, and lonis; B.L.M. reports honoraria from Biogen, Cambridge University Press Guilford Publications, Inc. Oxford University Press Taylor \& Francis Group Elsevier, Inc.; B.L.M. also served as medical/ scientific advisor of The John Douglas French Foundation, The Larry L. Hillblom Foundation, Association for Frontotemporal Degeneration, National Institute for Health Research Cambridge Biomedical Research Centre and its subunit, the Biomedical Research Unit in Dementia, University of Washington ADRC, Stanford University ADRC, Arizona Alzheimer's Disease Center (ADC), Massachusetts Alzheimer Disease Research Center, The Buck Institute for Research on Aging, University of Southern California (USC) PO1 Urban Air Pollution and Alzheimer's Disease: Risk, Heterogeneity and Mechanisms. A.Lleó has a patent (WO2019175379 A1 Markers of synaptopathy in neurodegenerative disease); received personal consulting fees from Biogen, Nutricia, Roche, and FujirebioEurope; and has served at scientific advisory boards from Fujirebio-Europe, Nutricia, Biogen, and Roche Diagnostics and has filed a patent application of synaptic markers in neurodegenerative diseases; M.G.T. reports no disclosures; R.S.V. has served at scientific advisory boards from Wave pharmaceuticals and lonis-Biogen and received personal honoraria from Janssen, Roche Diagnostics, Neuraxpharm for educational activities; D.C.P. reports no disclosures; J.F. has a patent (WO2019175379 A1 Markers of synaptopathy in neurodegenerative disease), has served at advisory boards for AC Immune, Zambon, and Lundbeck, and received honoraria for lectures from Roche, NovoNordisk, Esteve, and Biogen.

\section{Author details}

${ }^{1}$ Memory Unit, Department of Neurology, Hospital de la Santa Creu i Sant Pau, Biomedical Research Institute Sant Pau, Sant Antoni Maria Claret, 167, 08025 Barcelona, Spain. ${ }^{2}$ Centro de Investigación Biomédica en Red de Enfermedades Neurodegenerativas (CIBERNED), Barcelona, Spain. ${ }^{3}$ Atlantic Fellow for Equity in Brain Health at the University of California San Francisco, San Francisco, CA 94115, USA. ${ }^{4}$ Alzheimer's Disease and Other Cognitive Disorders Unit, Service of Neurology, Hospital Clínic de Barcelona, Institut d'Investigació Biomèdica August Pi i Sunyer, University of Barcelona, 08036 Barcelona, Spain. ${ }^{5}$ Memory and Aging Center, Department of Neurology, University of California, San Francisco, CA 94115, USA. ${ }^{6}$ Radiology Department, Hospital Clinic BarceIona and Magnetic Resonance Image Core facility, Institut d'Investigacions Biomèdiques August Pi i Sunyer (IDIBAPS), Barcelona, Spain. ${ }^{7}$ Department of Radiology, Hospital del Mar, Barcelona, Spain. ${ }^{8}$ Department of Neurology, Massachusetts General Hospital and Harvard Medical School, Boston, MA, USA. ${ }^{9}$ Massachusetts Alzheimer's Disease Research Center, Boston, MA, USA. ${ }^{10}$ Barcelona Down Medical Center. Fundació Catalana de Síndrome de Down, Barcelona, Spain.

\section{Received: 29 September 2021 Accepted: 2 February 2022} Published online: 09 February 2022

\section{References}

1. Mesulam MM. Primary progressive aphasia. Ann Neurol. 2001;49:425-32.

2. Elahi FM, Miller BL. A clinicopathological approach to the diagnosis of dementia. Nat Rev Neurol. 2017;13:457-76.

3. Gorno-Tempini ML, Hillis AE, Weintraub S, Kertesz A, Mendez M, Cappa SF, et al. Classification of primary progressive aphasia and its variants. Neurology. 2011;76:1006-14.

4. Tippett DC, Hillis AE, Tsapkini K. Treatment of primary progressive aphasia. Curr Treat Options Neurol. 2015;17:362.

5. Coyle-Gilchrist ITS, Dick KM, Patterson K, Vázquez Rodríquez P, Wehmann E, Wilcox A, et al. Prevalence, characteristics, and survival of frontotemporal lobar degeneration syndromes. Neurology. 2016;86:1736-43.

6. Mesulam MM, Wieneke C, Thompson C, Rogalski E, Weintraub S. Quantitative classification of primary progressive aphasia at early and mild impairment stages. Brain. 2012;135:1537-53.

7. Boxer AL, Gold M, Feldman H, Boeve BF, Dickinson SL-J, Fillit H, et al. New directions in clinical trials for frontotemporal lobar degeneration: methods and outcome measures. Alzheimers Dement. 2020;16:131-43. 
8. Rosen HJ, Boeve BF, Boxer AL. Tracking disease progression in familial and sporadic frontotemporal lobar degeneration: recent findings from ARTFL and LEFFTDS. Alzheimers Dement. 2020;16:71-8.

9. Brambati SM, Amici S, Racine CA, Neuhaus J, Miller Z, Ogar J, et al. Longitudinal gray matter contraction in three variants of primary progressive aphasia: a tenser-based morphometry study. Neurolmage Clin. 2015:8:345-55

10. Mandelli ML, Vilaplana E, Brown JA, Hubbard HI, Binney RJ, Attygalle S, et al. Healthy brain connectivity predicts atrophy progression in nonfluent variant of primary progressive aphasia. Brain. 2016;139:2778-91.

11. Collins JA, Montal V, Hochberg D, Quimby M, Mandelli ML, Makris N, et al. Focal temporal pole atrophy and network degeneration in semantic variant primary progressive aphasia. Brain. 2017;140:457-71.

12. Tetzloff KA, Duffy JR, Clark HM, Strand EA, Machulda MM, Schwarz CG, et al. Longitudinal structural and molecular neuroimaging in agrammatic primary progressive aphasia. Brain. 2017;141:302-17.

13. Weston PSJ, Simpson IJA, Ryan NS, Ourselin S, Fox NC. Diffusion imaging changes in grey matter in Alzheimer's disease: a potential marker of early neurodegeneration. Alzheimers Res Ther. 2015;7:47.

14. Montal V, Vilaplana E, Alcolea D, Pegueroles J, Pasternak O, Gonzalez-Ortiz $\mathrm{S}$, et al. Cortical microstructural changes along the Alzheimer's disease continuum. Alzheimers Dement. 2018;14:340-51.

15. Illan-Gala I, Montal V, Borrego-Ecija S, Vilaplana E, Pegueroles J, Alcolea D, et al. Cortical microstructure in the behavioural variant of frontotemporal dementia: looking beyond atrophy. Brain. 2019;142:1121-33.

16. Illán-Gala I, Montal V, Pegueroles J, Vilaplana E, Alcolea D, Dols-Icardo O, et al. Cortical microstructure in the amyotrophic lateral sclerosis-frontotemporal dementia continuum. Neurology. 2020;95:e2565-76.

17. Montal V, Vilaplana E, Pegueroles J, Bejanin A, Alcolea D, Carmona-Iragui $\mathrm{M}$, et al. Biphasic cortical macro- and microstructural changes in autosomal dominant Alzheimer's disease. Alzheimers Dement. 2021:17:618-28.

18. Vilaplana E, Rodriguez-Vieitez E, Ferreira D, Montal V, Almkvist O, Wall $A$, et al. Cortical microstructural correlates of astrocytosis in autosomal dominant Alzheimer disease. Neurology. 2020;10.1212/ WNL.0000000000009405.

19. Santos-Santos MA, Mandelli ML, Binney RJ, Ogar J, Wilson SM, Henry ML, et al. Features of patients with nonfluent/agrammatic primary progressive aphasia with underlying progressive supranuclear palsy pathology or corticobasal degeneration. JAMA Neurol. 2016;73:733-10.

20. Alcolea D, Clarimon J, Carmona-Iragui M, Illan-Gala I, Morenas-Rodriguez E, Barroeta I, et al. The Sant Pau Initiative on Neurodegeneration (SPIN) cohort: a data set for biomarker discovery and validation in neurodegenerative disorders. Alzheimers Dement (N Y). 2019;5:597-609.

21. Perry DC, Brown JA, Possin KL, Datta S, Trujillo A, Radke A, et al. Clinicopathological correlations in behavioural variant frontotemporal dementia. Brain. 2017:140:3329-45.

22. Mackenzie IRA, Neumann M, Bigio EH, Cairns NJ, Alafuzoff I, Kril J, et al. Nomenclature and nosology for neuropathologic subtypes of frontotemporal lobar degeneration: an update. Acta Neuropathol. 2010;119:1-4.

23. Ranasinghe KG, Rankin KP, Lobach IV, Kramer JH, Sturm VE, Bettcher BM, et al. Cognition and neuropsychiatry in behavioral variant frontotemporal dementia by disease stage. Neurology. 2016;86:600-10.

24. Whitwell JL, Jack CR, Boeve BF, Senjem ML, Baker M, Rademakers R, et al. Voxel-based morphometry patterns of atrophy in FTLD with mutations in MAPT or PGRN. Neurology. 2009;72:813-20.

25. Moore KM, Nicholas J, Grossman M, McMillan CT, Irwin DJ, Massimo L, et al. Age at symptom onset and death and disease duration in genetic frontotemporal dementia: an international retrospective cohort study. Lancet Neurol. 2020;19:145-56.

26. Saracino D, Ferrieux S, Noguès-Lassiaille $M$, Houot $M$, Funkiewiez $A$, Sellami L, et al. Primary progressive aphasia associated with GRN mutations: new insights into the non-amyloid logopenic variant. Neurology. 2021

27. Morris JC. The Clinical Dementia Rating (CDR): current version and scoring rules. Neurology. 1993;43:2412-4.

28. Miyagawa T, Brushaber D, Syrjanen J, Kremers W, Fields J, Forsberg LK, et al. Utility of the global CDR ${ }^{\circledast}$ plus NACC FTLD rating and development of scoring rules: data from the ARTFL/LEFFTDS Consortium. Alzheimers Dement. 2020;16:106-17.

29. Folstein MF, Folstein SE, McHugh PR. Mini-mental state. J Psychiatr Res. 1975:12:189-98
30. Calero O, Hortigüela R, Bullido MJ, Calero M. Apolipoprotein E genotyping method by real time PCR, a fast and cost-effective alternative to the TaqMan ${ }^{\circledR}$ and FRET assays. J Neurosci Methods. 2009;183:238-40.

31. Antonell A, Tort-Merino A, Ríos J, Balasa M, Borrego-Écija S, Auge JM, et al. Synaptic, axonal damage and inflammatory cerebrospinal fluid biomarkers in neurodegenerative dementias. Alzheimers Dement. 2020;16:262-72.

32. Hyman BT, Phelps CH, Beach TG, Bigio EH, Cairns NJ, Carrillo MC, et al. National Institute on Aging-Alzheimer's Association guidelines for the neuropathologic assessment of Alzheimer's disease. Alzheimer's Dementia. 2012;8:1-13.

33. Fischl B, Dale AM. Measuring the thickness of the human cerebral cortex from magnetic resonance images. Proc Natl Acad Sci. 2000;97:11050-5.

34. Hagler DJ, Saygin AP, Sereno MI. Smoothing and cluster thresholding for cortical surface-based group analysis of fMRI data. Neurolmage. 2006:33:1093-103.

35. Parker TD, Slattery CF, Zhang J, Nicholas JM, Paterson RW, Foulkes AJM, et al. Cortical microstructure in young onset Alzheimer's disease using neurite orientation dispersion and density imaging. Hum Brain Mapp. 2018;39:3005-17.

36. Ganepola T, Nagy Z, Ghosh A, Papadopoulo T, Alexander DC, Sereno MI. Using diffusion MRI to discriminate areas of cortical grey matter. Neuroimage. 2018;182:456-68.

37. Coalson TS, Van Essen DC, Glasser MF. The impact of traditional neuroimaging methods on the spatial localization of cortical areas. Proc Natl Acad Sci U S A. 2018;115:E6356-65.

38. Fortin J-P, Parker D, Tunç B, Watanabe T, Elliott MA, Ruparel K, et al. Harmonization of multi-site diffusion tensor imaging data. Neurolmage. 2017;161:149-70.

39. Whitwell JL, Avula R, Senjem ML, Kantarci K, Weigand SD, Samikoglu A, et al. Gray and white matter water diffusion in the syndromic variants of frontotemporal dementia. Neurology. 2010;74:1279-87.

40. Seeley WW, Crawford RK, Zhou J, Miller BL, Greicius MD. Neurodegenerative diseases target large-scale human brain networks. Neuron. 2009:62:42-52.

41. Knopman DS, Kramer JH, Boeve BF, Caselli RJ, Graff-Radford NR, Mendez $M$, et al. Development of methodology for conducting clinical trials in frontotemporal lobar degeneration. Brain. 2008;131:2957-68.

42. Spinelli EG, Mandelli ML, Miller ZA, Santos-Santos MA, Wilson SM, Agosta $F$, et al. Typical and atypical pathology in primary progressive aphasia variants. Ann Neurol. 2017;81:430-43.

43. Leyton CE, Hodges JR, McLean CA, Kril JJ, Piguet O, Ballard KJ. Is the logopenic-variant of primary progressive aphasia a unitary disorder? Cortex. 2015;67:122-33.

44. Pegueroles J, Vilaplana E, Montal V, Sampedro F, Alcolea D, CarmonaIragui $\mathrm{M}$, et al. Longitudinal brain structural changes in preclinical Alzheimer's disease. Alzheimers Dement. 2017;13:499-509.

45. Ossenkoppele R, Schonhaut DR, Schöll M, Lockhart SN, Ayakta N, Baker $\mathrm{SL}$, et al. Tau PET patterns mirror clinical and neuroanatomical variability in Alzheimer's disease. Brain. 2016;139:1551-67.

46. Downey LE, Mahoney CJ, Buckley AH, Golden HL, Henley SM, Schmitz N, et al. White matter tract signatures of impaired social cognition in frontotemporal lobar degeneration. Neurolmage Clin. 2015;8:640-51.

47. McMillan CT, Boyd C, Gross RG, Weinstein J, Firn K, Toledo JB, et al. Multimodal imaging evidence of pathology-mediated disease distribution in corticobasal syndrome. Neurology. 2016;87:1227-34.

48. Giannini LAA, Xie SX, McMillan CT, Liang M, Williams A, Jester C, et al. Divergent patterns of TDP-43 and tau pathologies in primary progressive aphasia. Ann Neurol. 2019;85:630-43.

49. Staffaroni AM, Ljubenkov PA, Kornak J, Cobigo Y, Datta S, Marx G, et al. Longitudinal multimodal imaging and clinical endpoints for frontotemporal dementia clinical trials. Brain. 2019;142:443-59.

50. Rohrer JD, Nicholas JM, Cash DM, van Swieten J, Dopper E, Jiskoot L, et al. Presymptomatic cognitive and neuroanatomical changes in genetic frontotemporal dementia in the Genetic Frontotemporal dementia Initiative (GENFI) study: a cross-sectional analysis. Lancet Neurol. 2015;14:253-62.

\section{Publisher's Note}

Springer Nature remains neutral with regard to jurisdictional claims in published maps and institutional affiliations. 